\title{
Zusammenhänge zwischen der Soziallage, Empowermentprozessen und der Entwicklung psychischer Gesundheit
}

\section{Correlation between Social Status, Empowerment Processes and the Development of Psychological Health}

\author{
Autor \\ S. Sperlich \\ Institut \\ Medizinische Soziologie, Medizinische Hochschule Hannover
}

\author{
Schlüsselwörter \\ - Evaluation \\ - Gesundheitliche \\ Ungleichheit \\ - Versorgungsforschung \\ - Mütter \\ - Empowerment
}

Key words
evaluation
health inequality
health-care research
mothers, empowerment

Bibliografie

DOI 10.1055/s-0028-1103263

Gesundheitswesen 2008;

70: 779-790

(c) Georg Thieme Verlag KG

Stuttgart · New York

ISSN 0941-3790

Korrespondenzadresse

Dr. S. Sperlich

Medizinische Hochschule

Hannover

Medizinische Soziologie

OE 5420

Carl-Neuberg-Str. 1

30625 Hannover

sperlich.stefanie@

mh-hannover.de

\section{Zusammenfassung}

$\nabla$

Die vorliegende Arbeit verfolgt die Zielsetzung, das Wissen über soziallagespezifische Empowermentprozesse anhand von katamnestischen Daten aus Vorsorge- und Rehabilitationseinrichtungen für Mütter und ihre Kinder zu fundieren. Im Mittelpunkt der Untersuchung steht die Beantwortung folgender Fragen: 1. Wie entwickelt sich die psychische Gesundheit in Abhängigkeit von der sozialen Lage nach der Intervention? 2. welche soziallagespezifischen Empowermentprozesse bilden sich poststationär ab? und 3 . welche Bedeutung kommt Empowerment für die nachhaltige Verbesserung der psychischen Symptombelastung zu? Die Analyse basiert auf Daten von 6094 Patientinnen aus 39 Vorsorge- und Rehabilitationseinrichtungen, die bis zu einem Jahr nach der Intervention erhoben wurden. Für die Evaluation von Empowerment wurden zwei Itembatterien entwickelt, die sich auf kleinräumige Veränderungen der Lebenssituation (,Verhältnis-Empowerment') sowie auf Optimierungen von gesundheitsbezogenen Verhaltensweisen, Einstellungen und Kompetenzen (,Verhaltens-Empowerment') beziehen. Veränderungen im Gesundheitszustand wurden hinsichtlich der Dimension ,psychische Symptombelastung، (SCL-K-9) erfasst. Die soziale Schichtzugehörigkeit wurde clusteranalytisch bestimmt, die soziallagespezifische Gesundheitsrelevanz von Empowerment regressionsanalytisch ermittelt. Die gesundheitlich am stärksten belasteten Mütter der unteren sozialen Lage profitieren kurzfristig am meisten von der Intervention, jedoch gehen die Therapieeffekte in dieser sowie in der mittleren Soziallage nach der Rückkehr in den häuslichen Alltag überdurchschnittlich stark zurück. Insbesondere für die Mütter der unteren und mittleren Soziallage erwies sich das ,Verhaltens-Empowerment' als bedeutsam für die nachhaltige Verbesserung des psychischen Gesund-

\section{Abstract \\ $\nabla$}

The aim of this paper is to deepen our knowledge about empirical evidence of empowerment on the basis of follow-up data from mother-child rehabilitation centres. The study focusses on three questions: i) to what extent can psychological health in different socioeconomic groups be improved by the intervention?, ii) Is there an impact of social position on the success of empowerment and iii) Is there any relevance of empowerment for sustainable improvement of psychological health? The study is based on data from 6095 patients of 39 mother-child rehabilitation centres in Germany, collected up to one year after intervention. Empowerment was assessed by two self-defined scales, measuring positive changes i) in living circumstances, such as partnership or household ('Verhältnis-Empowerment') and ii) in health-related behaviours and competencies, such as better coping with problems and higher health awareness ('Verhaltens-Empowerment'). Health status was assessed by psychological symptoms and measured by SCL-K-9, a short form of the SCL-90-R. The socioeconomic position was determined by cluster analysis, the influence of social position and empowerment for psychological health was computed by analysis of regression. Before intervention mothers from the lower class showed higher degrees of psychological symptoms. After intervention they reached higher short-term effects, but six months later psychological symptoms increased again. In particular improvement of health-related behaviours and competencies was associated with better psychological health. The highest impact on health was found among mothers from the middle and lower class. These mothers, however, showed less success in optimising health-related behaviours and competencies. Twelve months after treatment the health-related impact of empowerment decreased. The theoretical evidence 
heitszustandes, allerdings sind die Empowermenterfolge hier im Vergleich zu den Müttern der oberen Soziallage vor allem in der Katamnese nach sechs Monaten deutlich geringer. In der Katamnese nach zwölf Monaten ist ein deutlicher Rückgang in der Bedeutung von Empowerment zu verzeichnen, wobei die relative Bedeutung des Verhältnis-Empowerments in der langfristigen Perspektive zunimmt. Die theoretische Evidenz von Empowerment konnte empirisch bestätigt werden. Der Umstand, dass die Gesundheitseffekte zwölf Monate nach der Intervention deutlich zurückgehen, weist auf die Notwendigkeit von unterstützenden poststationären therapeutischen Maßnahmen hin. Die ermittelten geringen Unterschiede zwischen den Empowermenterfolgen der unteren und mittleren Soziallage legen nahe, dass sozialschichtspezifische Analysen im vorliegenden Fall nur begrenzt aussagekräftig sind.

\section{Einleitung \\ $\nabla$}

In den letzten Jahren haben Prävention und Gesundheitsförderung in Deutschland einen deutlichen Aufschwung erfahren [1]. Zeitgleich avancierte der Begriff des Empowerments zum Leitgedanken einer auf die Stärkung der Eigenverantwortlichkeit und Selbstbefähigung setzenden Präventionsstrategie. Angesichts der besonderen Gesundheitsrisiken bei sozial benachteiligten Bevölkerungsgruppen hat Empowerment vor allem als Interventionsziel für die Verringerung gesundheitlicher Ungleichheit zunehmende Bedeutung erlangt. In diesem Kontext lautet die Zielsetzung, sozial benachteiligte Menschen zu befähigen, in ihrem Alltag gesundheitsförderliche Lebensstrukturen aufzubauen und ihr Leben gesundheitsbewusster und gesundheitsgerechter zu gestalten [2,3]. In jüngerer Zeit lässt sich eine verstärkte Qualitätssicherung und Dokumentationsqualität der Interventionsmaßnahmen zur Verringerung gesundheitlicher Ungleichheit beobachten. Von den in den letzten Jahren vollzogenen Qualitätsentwicklungen zeugt die bundesweite Datenbank ,Gesundheitsförderung bei sozial Benachteiligten', aus der erstmals Beispiele über ,good-practice‘ ableitbar sind. Empowerment im Sinne einer „Befähigung und Qualifizierung der Zielgruppe sozial Benachteiligter, die auf den Stärken und Ressourcen der Zielgruppen aufbaut" stellt dabei explizit eines der zwölf Kriterien zur Bewertung von guten Praxismodellen dar [1, S. 20].

Gleichwohl der Begriff des Empowements Eingang in nahezu alle Konzepte der Gesundheitsförderung gefunden hat, werden die dem Begriff zugrunde liegenden Interventionsziele häufig nicht konkretisiert. Empowerment wird damit, ähnlich wie der Begriff der Salutogenese, bislang vorwiegend zur allgemeinen Charakterisierung eines ressourcenorientierten Bezugsrahmens für Interventionsansätze verwendet. Evaluationsstudien zur empirischen Überprüfung der Zielerreichung von Empowerment sowie seiner Gesundheitsrelevanz stehen bislang noch weitgehend aus. Dies hat zur Folge, dass die empirische Evidenz des Empowerment-Konstruktes deutlich hinter der großen theoretischen Bedeutung des Modells zurückliegt [4,5]. In der Literatur werden vor allem methodische Schwierigkeiten als zentrales Hindernis einer dem Grundsatz nach geteilten Notwendigkeit der Wirksamkeitsprüfung von Empowerment benannt. So lässt sich die Zielerreichung von Empowerment weitaus schwieriger evaluieren, als der klassische ,Vorher-Nachher-Vergleich' des Gesundheitsstatus [6,7]. Da sich Empowerment-Entwicklungen erst mit zeitlicher Verzögerung zum Interventionsende abzeichnen, ist für die Empowerment-Evaluation zudem eine zeit- und of empowerment could be empirically confirmed. However, decreasing health effects of empowerment after twelve months show that supporting help after clinical intervention is necessary to maintain the positive impact of empowerment. Only small differences in empowerment between lower and middle class lead to the conclusion that terms of social class are less appropriate for the clinical sample of mothers investigated in this study.

kostenaufwendige katamnestische Nachbefragung erforderlich. In konzeptioneller Hinsicht besteht noch erheblicher Entwicklungsbedarf hinsichtlich der Operationalisierung von Empowermentdimensionen. Für den Bereich der Gesundheitsförderung liegen zwar allgemeine Instrumente zur Erfassung von Veränderungen von gesundheitsrelevantem Wissen, Einstellungen und Verhalten vor (z.B. [8]), validierte settingspezifische Empowermentinstrumente sind bislang jedoch noch kaum entwickelt worden.

Die bislang vorliegenden Befunde über die Implementierung und den Erfolg von Empowermentprozessen weisen auf erschwerte Empowermentbedingungen in den unteren Soziallagen hin. In diesem Zusammenhang wird vor allem angeführt, dass Menschen aus privilegierten Lebenslagen eher die Chance haben, ihr Gesundheitspotenzial optimal auszubilden $[9,10]$. Die individuellen Möglichkeiten des Aufbaus von personalen Ressourcen und Kompetenzen sowie die positive Einflussnahme auf die kleinräumigen Lebensbedingungen stellen sich damit als ein sozial ungleich verteiltes Gut dar. Aus der Gesundheitsförderung ist insgesamt bekannt, dass präventive Appelle und Strategien (z.B. in Bezug auf die Teilnahme an Krebsfrüherkennungsuntersuchungen) in größerem Maße von Angehörigen der Mittel- und Oberschicht umgesetzt werden [11]. In gleicher Weise stellt Pflaumer [12] fest, dass die Prognosen für Empowerment gerade dort weniger günstig ausfallen, wo akuter Handlungsbedarf herrscht. Die bislang umfassendste internationale Übersicht über die gesundheitsbezogene Effektivität von Empowerment in seinen unterschiedlichen Bedeutungskontexten (psychologisches und politisches Empowerment) liefert das WHO-Regionalbüro für Europa [13]. Insgesamt lässt sich noch ein erheblicher Forschungsbedarf konstatieren. Bislang erst unzureichend beantwortet ist beispielsweise die Frage, ob sich die hohe theoretische Evidenz von Empowerment als effektive gesundheitsförderliche Strategie auch empirisch bestätigen lässt und welche Bedingungen und Prozesse Empowermententwicklungen begünstigen bzw. erschweren.

\section{Empowerment in Vorsorge- und}

Rehabilitationseinrichtungen für Mütter/Väter und ihre Kinder

Vorsorge- und Rehabilitationseinrichtungen für Mütter/Väter und ihre Kinder richten sich prinzipiell an beide Elternteile, überwiegend wird die Maßnahme jedoch von Müttern mit Erschöpfungszuständen und multiplen somatischen und psychischen Beschwerden in Anspruch genommen [14]. Aus der Li- 
teratur bekannt ist, dass der Anteil kinderreicher Mütter, allein Erziehender sowie geschiedener bzw. in aktueller Trennungssituation befindlicher Mütter in der Maßnahme deutlich überdurchschnittlich ist. Die Patientinnen weisen insgesamt hohe psychosoziale Belastungen auf, insbesondere bedingt durch familiäre Überlastungen und finanzielle Problemlagen [14-16]. Mit ihrem sekundär-präventiven Ansatz unterscheiden sich die Einrichtungen in der Indikationsstellung deutlich von final orientierten Rehabilitationseinrichtungen. Differenzierungsmerkmale zu anderen Rehabilitationseinrichtungen sind vor allem der große Stellenwert psychosozialer Angebote und die therapeutische Integration der Kinder sowie der Mutter-Kind-Interaktion. Der Therapieansatz beruht auf einem ganzheitlichen Konzept, in dem physiotherapeutische, medizinische und psychosoziale Therapiebausteine ineinander greifen [15]. Neben indikationsspezifischen Behandlungsprogrammen, beispielsweise für die Diagnosen ,Psychische und Verhaltensstörungen“ oder ,Adipositas‘ bieten die Einrichtungen auch indikationsübergreifende Angebote im Sinne des Empowerments an. Die therapeutischen Bemühungen zielen hier zum einen auf die Reduzierung psychosozialer Belastungen durch die Veränderung der kleinräumigen Lebenssituation, vor allem familiärer Strukturen, wie die Beziehung zum Kind, die partnerschaftliche Situation oder die Organisation des Haushalts. Hierzu werden im Rahmen der sozialtherapeutischen Intervention Belastungssituationen aufgearbeitet und Handlungsstrategien zur positiven Veränderung des Alltags entwickelt. Zusätzliche präventive Angebote, wie Einzel- und Gruppenangebote zum Erlernen von Entspannungs- und Stressbewältigungsstrategien, GesundheitsVorträge und Erziehungskompetenztrainings dienen der subjektiven Kompetenz- und Ressourcenerweiterung, um potenziellen psychosozialen Belastungen adäquater begegnen zu können und damit das Risiko gesundheitlicher Folgen zu minimieren [17]. Die vorliegende Arbeit verfolgt das Ziel, anhand der klinischen Daten aus Vorsorge- und Rehabilitationseinrichtungen für Mütter und ihre Kinder das Wissen über soziallagespezifische Therapieeffekte und Empowermenterfolge zu vertiefen. Da die Maßnahme als besondere Form der medizinischen Vorsorge und Rehabilitation mit den Paragrafen 24 und 41 SGB V im Vertragsund Leistungsrecht der gesetzlichen Krankenversicherung verankert ist, lassen sich aus den Ergebnissen auch Aussagen über die Möglichkeiten der Reduzierung gesundheitlicher Ungleichheit im Rahmen des bestehenden Gesundheitssystems treffen. Damit leistet die vorliegende Untersuchung zugleich einen Beitrag zur Versorgungsforschung, die sich in den letzten Jahren zunehmend der gesundheitlichen Ungleichheitsthematik angenommen hat [18]. Im Mittelpunkt der vorliegenden Untersuchung steht die Beantwortung folgender drei Untersuchungsfragen:
- Wie entwickelt sich die psychische Gesundheit in Abhängigkeit von der sozialen Lage nach der Maßnahme?,

- Zeigen sich soziallagespezifische Unterschiede im ,Empowerment-Erfolg،, d.h. darin, wie es den Müttern nach der Maßnahme gelingt, ihre Lebenssituation und ihr Verhalten gesundheitsförderlicher zu gestalten? und

- Welche Bedeutung kommt Empowerment für die nachhaltige Verbesserung der psychischen Symptombelastung zu?

\section{Methodik \\ $\nabla$}

\section{Datenbasis}

Für die vorliegende Untersuchung werden Daten aus insgesamt 39 Präventions- und Rehabilitationseinrichtungen für Mütter und ihre Kinder aus dem gesamten Bundesgebiet reanalysiert. Sie stammen aus einer prospektiven Verlaufsstudie mit vier Erhebungszeitpunkten t1 (Beginn der Maßnahme), t2 (Ende der Maßnahme), t3 (sechs Monate nach der Maßnahme) sowie t4 (zwölf Monate nach der Maßnahme). Die Datenerhebung fand in den Jahren 2000-2003 statt, die Katamnese erstreckte sich bis 2004. Die Stichprobengröße der Haupterhebung zu t1 und t 2 beläuft sich auf 6094 Patientinnen und 8550 Kinder. In die Katamnese nach sechs bzw. zwölf Monaten wurde eine Teilpopulation von 4837 Patientinnen einbezogen. Die Rücklaufquote der postalischen Nachbefragung betrug nach sechs Monaten (t3) 67,6\% ( $n=3271)$ bzw. nach zwölf Monaten (t4) 50\% n=2425). Stratifiziert nach den drei im Rahmen dieser Arbeit fokussierten Soziallagen zeigt sich, dass die Mütter der unteren Soziallage sowohl an der Katamnese nach sechs als auch nach zwölf Monaten signifikant seltener teilgenommen haben ( $\bullet$ Tab. 1 ).

\section{Erhebungsinstrumente}

Psychische Gesundheit

Die psychische Gesundheit der Mütter wird mittels der SCL-K-9 erfasst. Die SCL-K-9 ist eine Kurzform der SCL-90-R [19] und erfasst mit 9 Items das Ausmaß psychischer Probleme in den letzten 7 Tagen. Es handelt sich dabei um folgende Symptome: ,Gefühlsausbrüche‘, ,dem Gefühl, dass es schwer fällt, etwas anzufangen', ,dem Gefühl, sich zu viele Sorgen zu machen', ,Verletzlichkeit in Gefühlsdingen“, ,dem Gefühl, dass man beobachtet wird‘, ,dem Gefühl, gespannt oder aufgeregt zu sein', ,Schweregefühl in Armen und Beinen', ,Nervosität, wenn man alleine ist und ,Einsamkeitsgefühle‘. Aus der Summe der Einzelitems wird ein Mittelwert gebildet mit einem Range von 0 ,überhaupt nicht bis 4 ,sehr stark'. Diese Gesamtskala gibt Auskunft über die allgemeine psychische Symptombelastung. Die SCL-K-9 ist reliabel (Chronbachs Alpha $=0,87$ ) und die Korrelation zur Langversion beträgt 0,93 [20]. Die SCL-K-9 wurde zu den Zeitpunkten t1 bis

Tab. 1 Fallzahlen der Haupterhebung (t1/t2) und Rücklaufrate der Katamnese (t3/t4) in den drei Soziallagen (in \%).

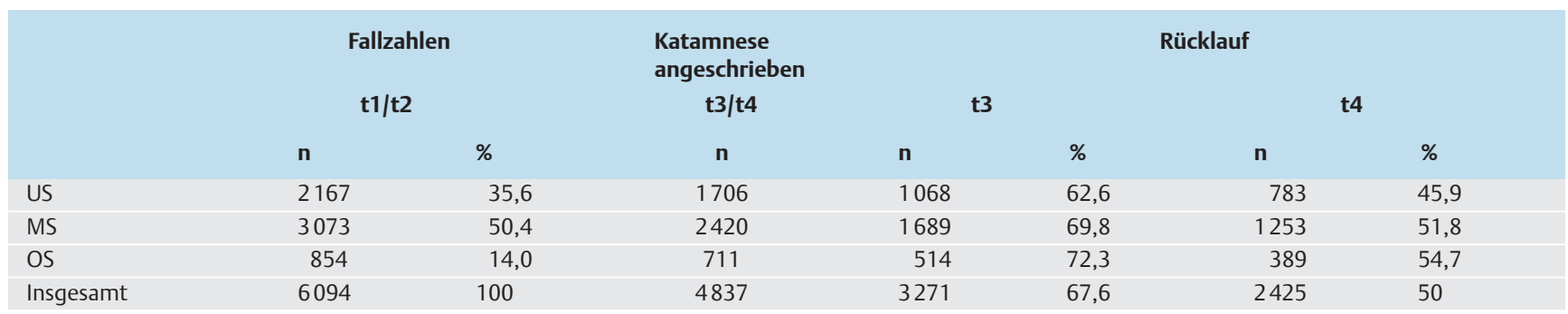

Erläuterungen: US= untere Soziallage, MS = mittlere Soziallage, OS= obere Soziallage. In die Katamnese -Erhebung t3 und t4 wurden nicht alle Patienten, sondern nur eine Zufallsstichprobe $(n=4837)$ einbezogen. 
t4 erhoben, wobei die Daten zu t3 und t4 nur von einer Teilstichprobe mit $n=1577$ (t3) bzw. $n=1134$ (t4) vorliegen.

\section{Empowerment}

Zur Messung von Empowermentprozessen wurden in Anlehnung an den Terminus aus der Prävention zwei Itembatterien zum ,Verhältnis-' und ,Verhaltens-Empowerment' entwickelt, die speziell den Empowermentbemühungen in Vorsorge- und Rehabilitationseinrichtungen Rechnung tragen (s. o.). Die Instrumentenentwicklung erfolgte im Rahmen des Forschungsverbundes ,Prävention und Rehabilitation für Mütter und ihre Kinder‘, der sich im Jahr 1997 als Zusammenschluss von Vorsorgeund Rehabilitations-Einrichtungen und einem Forschungsteam der Medizinischen Soziologie an der Medizinischen Hochschule zum Zwecke der internen und externen Qualitätssicherung konstituiert hat. In regelmäßig tagenden Qualitätszirkeln mit den Einrichtungsmitarbeiterinnen wurden auf der Basis der therapeutischen Einrichtungskonzeptionen die Empowerment-Instrumente entwickelt, deren Eignung getestet und im Rahmen von externen Evaluationen in den Einrichtungen eingesetzt. In die Instrumentenentwicklung eingeflossen sind zudem die Ergebnisse einer wissenschaftlichen Begleitforschung zu einem Modellversuch, der auf die Verbesserung von Vorsorge- und Rehabilitationseinrichtungen durch eine intensivierte Vor- und Nachbereitung zielte [21].

Beide Empowerment-Instrumente wurden sechs Monate (Katamnese t3) bzw. zwölf Monate (Katamnese t4) nach der Maßnahme im Rahmen eines umfangreichen Nacherhebungs-Fragebogens eingesetzt. Entsprechend beziehen sich die Empowermententwicklungen in der Katamnese zu t3 auf die letzten sechs Monate, zu t4 auf die letzten zwölf Monate nach der stationären Maßnahme.

Die Itembatterie zur Erfassung des Verhältnis-Empowerments bezieht sich mit insgesamt sechs fünfstufigen Items auf positive Veränderungen in unterschiedlichen kleinräumigen Lebensbereichen, die im Anschluss an die stationäre Maßnahme vollzogen wurden. Die Instruktion für die rückblickende Bewertung von Empowermentprozessen lautete folgendermaßen: „Nun würden wir gerne erfahren, welche Anregungen und Pläne Sie aus der Kur mit nach Hause genommen haben und was daraus geworden ist. Haben Sie nach der Kur Versuche unternommen, Ihre Lebenssituation zu verändern? Wenn ja, welche Ziele sind Sie angegangen? Wir haben im Folgenden mögliche Ziele aufgeführt. Kreuzen Sie bitte die auf Sie zutreffenden Ziele an und schildern Sie dazu kurz, was Sie unternommen haben. Geben Sie bitte zudem auf der Skala (von ,überhaupt nicht erfolgreich“ bis ,sehr erfolgreich') an, wie erfolgreich Sie ihre Veränderungsbemühungen beurteilen“. Im einzelnen wurden folgende Lebensbereiche vorgegeben: ,Partnerschaft‘, „Haushalt‘, ,Beziehung zum Kind', ,sonstige familiäre Beziehungen' (außer Partner und Kind), ,Beruf', ,Haushalt' und ,Freizeit‘. Die vorliegende Analyse bezieht sich ausschließlich auf die Erfolgseinschätzung der sechs vorgegebenen Lebensbereiche. Eine Analyse der Klartextangaben zu den konkreten Veränderungsbemühungen findet sich bei Sperlich [22].

Im Rahmen des Verhaltens-Empowerments wurden mit insgesamt zehn Items Aussagen erhoben, die Auskunft über die durch die Maßnahme initiierten Veränderungen gesundheitsrelevanter Verhaltensweisen, Einstellungen und Kompetenzen geben. Die Instruktion zu dieser Itembatterie lautete folgendermaßen: „Haben Sie durch Ihre Kur Anstöße erhalten und folgende Verhaltensweisen und Einstellungen verändert?" (Itemauflistung siehe unteren Teil $\bullet$ Tab. 4). Die Patientinnen wurden gebeten, den Grad der Zustimmung zu den einzelnen Aussagen auf einer fünfstufigen Skala (von 1 ,trifft überhaupt nicht zu' bis 5 ,trifft voll und ganz $\mathrm{zu}^{\prime}$ ) zu beurteilen.

Für die Analyse der grundsätzlichen Bedeutung von Empowermentprozessen wurden durch Summation der Einzelitems zwei Indikatorvariablen zum Verhältnis- und Verhaltens-Empowerment gebildet, die Auskunft über die durchschnittliche Erfolgseinschätzung bzw. die durchschnittliche Zustimmung über alle Einzelitems des Empowerments geben [22].

\section{Statistische Methoden}

\section{Bestimmung der sozialen Lage der Mütter}

Vor dem Hintergrund, dass die Lebenssituation von Frauen im besonderen Maße durch die Verknüpfung von klassischen Ungleichheitsdimensionen (Beruf, Bildung und Einkommen) und weiterführenden Ungleichheitsdimensionen (v.a. Lebensform, familiäre Situation, psychosoziale Stressoren) geprägt ist [2325], erfolgte die Bestimmung der sozialen Lage clusteranalytisch [26]. Dieses Verfahren gestattet es, neben den klassischen Ungleichheitsdimensionen auch weiterführende Variablen, insbesondere der familiären und psychosozialen Situation in die Soziallagebestimmung einfließen zu lassen. Aus den unterschiedlichen Verfahren der Clusteranalyse wurde das Quick-Clustering als eine Modifikation des K-Means-Verfahrens ausgewählt und mit der Prozedur „Quick Cluster“ berechnet. Bei den K-MeansVerfahren werden Clusterzentren zur Bildung der Cluster konstruiert. Die Clusterzentren für die Cluster werden so berechnet werden, dass die Streuungsquadratsumme in den Clustern ein Minimum ist [26]. Die Clusterbildung erfolgt nicht nach dem sehr rechenaufwändigen hierarchischen, sondern nach einem partitionierenden Algorithmus. Aus diesem Grund eignen sich die K-Means-Verfahren insbesondere für größere Datenmengen, wie sie hier vorliegen. Der Nachteil dieses Verfahrens ist, dass die Clusterzahl a priori vorgegeben werden muss. Um die optimale Clusterzahl zu bestimmen, wurde zunächst anhand einer $20 \%$-Stichprobe eine hierarchische Clusteranalyse durchgeführt. Die optimale Clusterzahl wurde aus den Fehlerquadratsummen (Abweichungen aller Variablenwerte zu den betreffenden Clustermittelwerten in allen Clustern) abgeleitet.

In Anlehnung an das Vorgehen von Babitsch [27] wurden aus den initial ermittelten sieben Lebenslagen drei übergeordnete Soziallagen gebildet, die in grober Form die drei Statuspositionen untere, mittlere und obere Sozialschicht repräsentieren. Ausschlaggebend für die inhaltliche Zuordnung zu den drei übergeordneten Schichtlagen war eine qualitative Analyse der Clustermittelwerte zu den klassischen Ungleichheitsdimensionen Schul- und Berufsbildung, finanzielle Situation und berufliche Stellung. Wenn mindestens eines dieser Kriterien einen deutlich unterdurchschnittlichen Wert aufwies, wurde das Cluster der unteren sozialen Lage zugeordnet. Lagen die Werte zumeist im mittleren Bereich (z. B. überdurchschnittlich häufig Realschulabschluss oder mittlere Berufsposition) und lagen keine ausgeprägten finanziellen Benachteiligungen vor, wurden die Cluster der mittleren sozialen Lage zugeordnet. Eine deutliche Besserstellung bezüglich aller drei Statuskriterien war das Kriterium für die Zuordnung zur oberen Soziallage.

Mit diesem Design ist es möglich, sowohl der gebotenen sozialen Differenzierung Rechnung zu tragen (Analyse der sieben Soziallagen) als auch strukturell verursachter gesundheitlicher Benachteiligung nachzugehen (Analyse der übergeordneten Sozialschichten). Die folgenden Analysen beziehen sich ausschließlich 
Tab. 2 Häufigkeitsverteilung der sozialen Charakteristika in den drei Soziallagen (in \%).

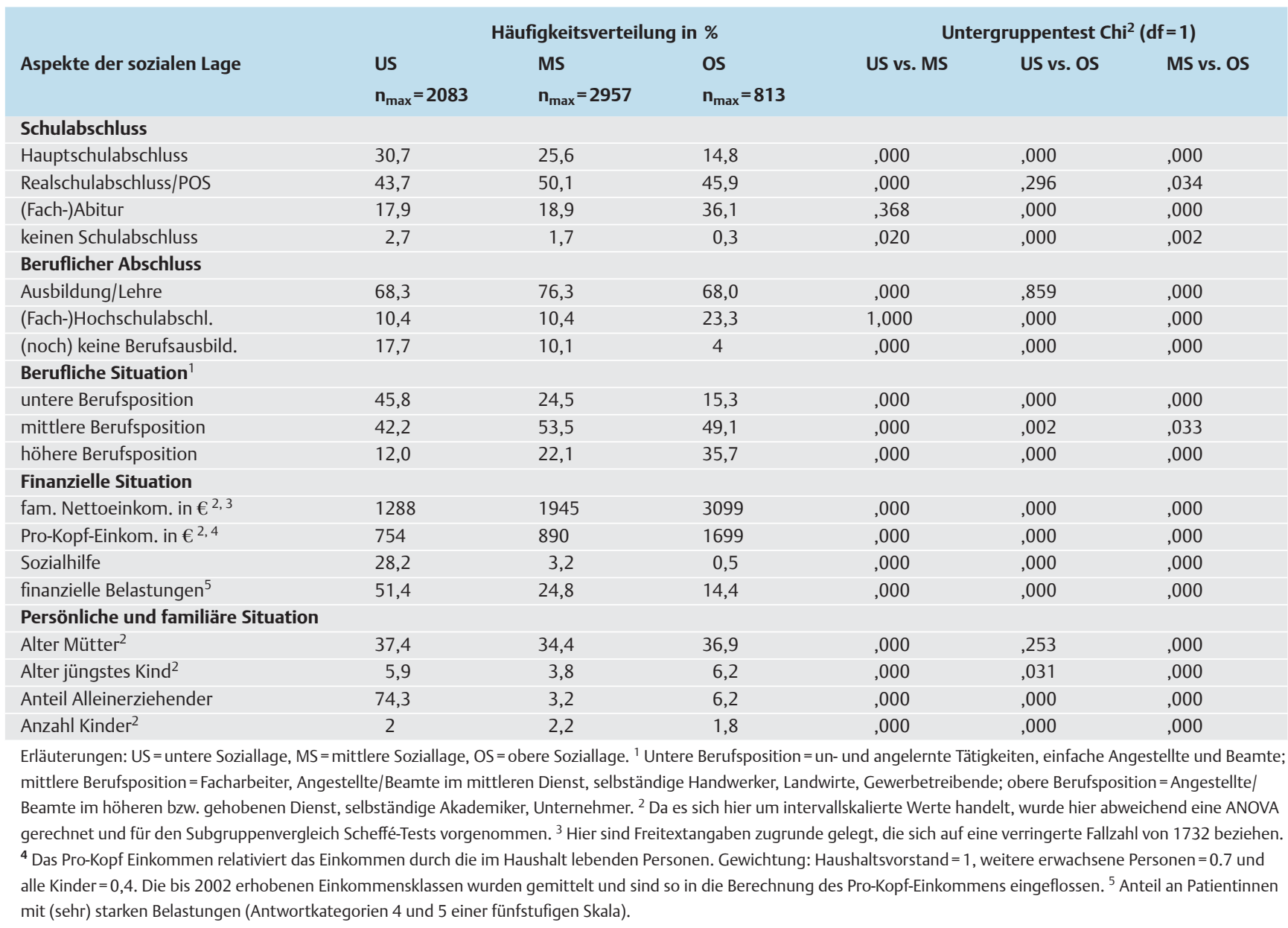

auf die drei übergeordneten Soziallagen. Dies entspricht der Zielsetzung, die Entwicklung von psychischer Gesundheit und Empowermentprozessen zunächst anhand der klassischen Indikatoren sozialer Ungleichheit zu analysieren. Die Validität der drei übergeordneten Soziallagen wurde mittels paarweise $\mathrm{Chi}^{2}$ Tests nach Pearson gemessen. Die Validitätsprüfung zeugt insgesamt von einer hohen internen Validität der drei Schichtlagen. Damit kann die qualitative Zuordnung zu den drei übergeordneten Sozialschichten mit wenigen Einschränkungen als valide bezeichnet werden ( $\boldsymbol{\Theta}$ Tab. 2 ).

\section{Evaluation der soziallagespezifischen Therapieeffekte und Empowermenterfolge}

Bezüglich der psychischen Symptombelastung liegen Längsschnittdaten von $\mathrm{t} 1$ bis $\mathrm{t} 4$ vor, die eine indirekte (Prä-Post-) Veränderungsmessung ermöglichen. Zur Darstellung der sozialschichtspezifischen kurz-, mittel- und langfristigen Therapieerfolge wurden sogenannte Effektstärken (SES) berechnet. Die Effektstärke berechnet sich als Mittelwertdifferenz von Prä- und Postwerten, standardisiert an der Standardabweichung der Messwerte vor der Maßnahme (t1). Effektstärken ab 0,2 gelten als gering, ab 0,5 als mittel und ab 0,8 als hoch [28]. Die retrospektiv zu t3 bzw. t4 erhobenen Empowermententwicklungen wurden zunächst deskriptiv durch einfache Häufigkeitsauszählungen und Kreuztabellierungen mit den Soziallagen erfasst. Zur Bestimmung, welche Soziallagen sich hinsichtlich des Empowermenterfolges signifikant unterscheiden, wurden $\mathrm{Chi}^{2}$-Tests nach Pearson durchgeführt.

\section{Einfluss von Empowerment auf die Entwicklung der} poststationären psychischen Gesundheit

Um die soziallagespezifische Bedeutung von Empowerment auf die poststationäre Entwicklung des Gesundheitszustandes zu analysieren, wurden gesondert für die Mütter der oberen, mittleren und unteren Sozialschicht und getrennt für den Zeitraum t 3 und t 4 multiple lineare Regressionsmodelle gerechnet. Als abhängige Variable ist jeweils die psychische Symptombelastung zu t3 (Regression I) bzw. t4 (Regression II) in die Analyse eingegangen. Ausgehend von der Annahme, dass auch die vorangegangene psychische Symptombelastung das Ausmaß der aktuellen Symptombelastung vorhersagt, wurden als Prädiktoren in dem ersten Block zunächst die psychischen Symptombelastungen zu den vorangegangenen Untersuchungszeitpunkten in das Modell aufgenommen. Getrennt voneinander wurden anschließend die Indikatorvariablen zum Verhältnis-Empowerment (Block 2) und Verhaltens-Empowerment (Block 3) integriert. Zur Ermittlung, welchen Empowerment-Aspekten auf Itemebene besondere Gesundheitsrelevanz zukommt, wurden (kontrolliert für die vorausgegangene psychische Symptombelastung) einfache Regressionsmodelle gerechnet. Die abhängige Variable stellte auch hier die poststationäre Symptombelastung zu t3 bzw. t4 dar, als Prädiktoren sind jeweils die Einzelitems des Empowements eingegangen.

\section{Stichprobencharakterisierung}

Die sozialen und familiären Charakteristika der Patientinnen der oberen, mittleren und unteren Soziallage sind der 0 Tab. 2 zu 


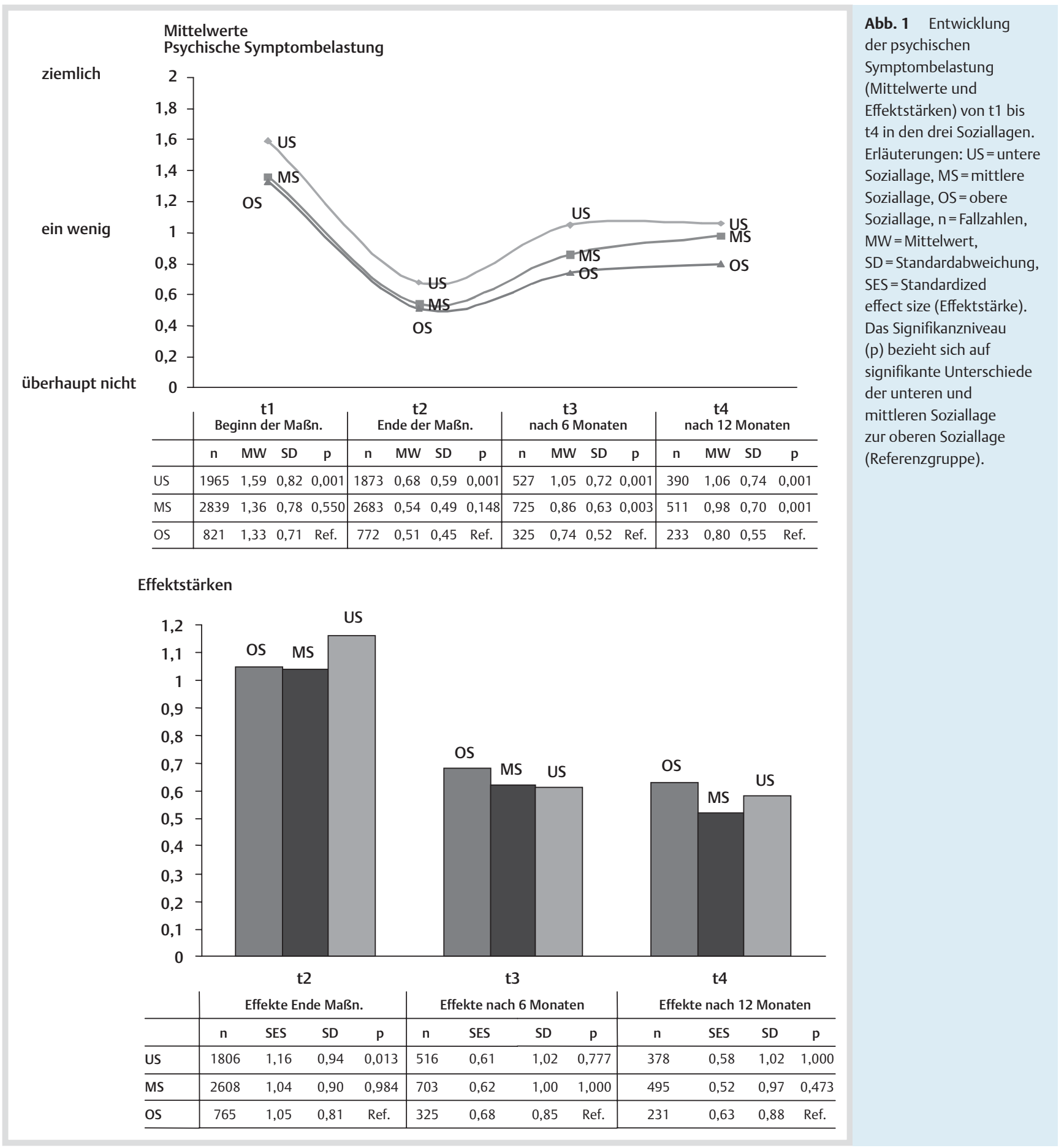

entnehmen. Aus ihr ersichtlich werden die Unterschiede zwischen den sozioökonomischen Indikatoren der oberen, mittleren und unteren Soziallage. Hinsichtlich der persönlichen und familiären Situation zeigt sich, dass in der mittleren Soziallage sowohl die jüngsten Mütter als auch die jüngsten Kinder anzutreffen sind. Gleichzeitig findet sich hier die durchschnittlich höchste Kinderzahl. In der unteren Soziallage finden sich mit einem Anteil von 74\% überwiegend alleinerziehende Mütter.

\section{Ergebnisse}

Sozialschichtspezifische kurz-, mittel- und langfristige Therapieeffekte

$\mathrm{Zu}$ Beginn der Maßnahme weisen die Frauen der unteren gegenüber der oberen Soziallage eine signifikant höhere psychische Symptombelastungn auf. Zum Ende der dreiwöchigen Maßnahme zeigt sich soziallageübergreifend eine deutliche Verbesserung der psychischen Gesundheit, die gemäß des größten Veränderungspotenzials in der unteren Soziallage besonders hoch ausfällt ( $\bullet$ Abb. 1). In der Konsequenz verringern sich zum Ende der Maßnahme die gesundheitlichen Unterschiede zwischen den Soziallagen. Nach wie vor weisen die Mütter der unteren 
Tab. 3 Soziallagespezifische Umsetzung des Verhältnis-Empowerments (in \%) zu t3/t4.

\begin{tabular}{|c|c|c|c|c|c|c|c|c|}
\hline \multirow[b]{2}{*}{ Anzahl Veränderungen } & \multicolumn{3}{|c|}{ Katamnese $\mathbf{t 3}$} & & \multicolumn{3}{|c|}{ Katamnese t4 } & \multirow[b]{2}{*}{$\mathrm{p}\left(\mathrm{Chi}^{2}\right)$} \\
\hline & $\begin{array}{l}\text { US } \\
n=1070\end{array}$ & $\begin{array}{l}\text { MS } \\
n=1689\end{array}$ & $\begin{array}{l}\text { OS } \\
n=514\end{array}$ & $\mathrm{p}\left(\mathrm{Chi}^{2}\right)$ & $\begin{array}{l}\text { US } \\
n=783\end{array}$ & $\begin{array}{l}\text { MS } \\
n=1253\end{array}$ & $\begin{array}{l}\text { OS } \\
n=389\end{array}$ & \\
\hline keine & 20,8 & 27,8 & 24,1 & \multirow[t]{4}{*}{$<, 001$} & 4,6 & 6,3 & 4,6 & \multirow[t]{4}{*}{, 443} \\
\hline eine & 10,3 & 8,5 & 11,1 & & 7,2 & 7 & 9 & \\
\hline zwei & 15,9 & 16,9 & 19,8 & & 11,4 & 12,5 & 11,6 & \\
\hline drei und mehr & 53 & 46,8 & 44,9 & & 76,9 & 74,2 & 74,8 & \\
\hline \multicolumn{9}{|l|}{ Lebensbereiche } \\
\hline Partnerschaft & 43,4 & 38,8 & 35 &, 004 & 58,4 & 64,3 & 56 &, 002 \\
\hline Beziehung Kind & 55,9 & 50,3 & 46,3 &, 001 & 78,8 & 75,2 & 73 &, 057 \\
\hline fam. Beziehungen & 32,7 & 26,9 & 23,2 & $<, 001$ & 64,1 & 58,7 & 54,2 &, 003 \\
\hline berufliche Sit. & 37,9 & 31,9 & 33,3 &, 005 & 65,4 & 60,6 & 61,7 & ,089 \\
\hline Haushalt & 37,5 & 36,7 & 34,6 &, 543 & 65,3 & 62,3 & 60,4 & ,215 \\
\hline Freizeit & 52 & 47,2 & 48,4 & ,049 & 73,1 & 73,3 & 69,7 & ,348 \\
\hline
\end{tabular}

Erläuterungen: US = untere Soziallage, MS = mittlere Soziallage, OS= obere Soziallage.

Soziallage jedoch noch die höchsten psychischen Beeinträchtigungen auf. Die Therapieeffekte in der unteren Soziallage gehen sechs Monate nach der Maßnahme überdurchschnittlich stark zurück. Dies dokumentiert sich in den zu t3 nunmehr kleinsten erzielten Effektstärken und einer relativen Verschlechterung im Gesundheitszustand im Vergleich zu den Müttern der oberen Soziallage. Auch die Therapieeffekte der Mütter der mittleren Soziallage gehen zu t3 im Vergleich zur oberen Soziallage überdurchschnittlich stark zurück. In der Folge zeigt sich gegenüber dem Zeitpunkt vor der Maßnahme (t1) eine Vergrößerung gesundheitlicher Ungleichheit zwischen den Müttern der unteren und mittleren Soziallage auf der einen und den Müttern der oberen Soziallage auf der anderen Seite. In der Katamnese nach zwölf Monaten verändert sich die psychische Symptombelastung in der unteren Soziallage nur geringfügig, während sie in der oberen Soziallage moderat und in der mittleren Soziallage deutlich ansteigt. Die höchsten Effektstärken nach zwölf Monaten und damit den größten langfristigen Therapieerfolg weisen die Mütter der oberen Soziallage auf. Die Effektstärken unterscheiden sich jedoch nur tendenziell und nicht signifikant zwischen den Soziallagen. Grundsätzlich lässt sich festhalten, dass alle Frauen von der Maßnahme profitieren konnten und noch zwölf Monate nach der Intervention signifikante Verbesserungen der psychischen Gesundheit aufweisen.

\section{Sozialschichtspezifische Empowermentprozesse}

Absichten zur Veränderung der Lebenssituation und ihre tatsächliche Umsetzung (Verhältnis-Empowerment)

Am Ende der dreiwöchigen stationären Maßnahme beabsichtigen $80,6 \%$ der Mütter der unteren gegenüber $79,1 \%$ der mittleren und $76,9 \%$ der oberen Soziallage, nach der Rückkehr nach Hause Veränderungen der kleinräumigen Lebenssituation vorzunehmen (ohne Darstellung). Tendenziell bekunden damit Mütter der unteren Sozialschicht eine größere Veränderungsbereitschaft, die Unterschiede zwischen den Soziallagen fallen jedoch nicht signifikant aus. In der Katamnese nach sechs Monaten (t3) zeigt sich, dass die Mütter der unteren Sozialschicht auch signifikant häufiger Veränderungen ihrer Lebenssituation vollzogen haben ( $\bullet$ Tab. 3 ). In der Katamnese nach zwölf Monaten ( $t 4$ ) geben fast alle Mütter mindestens eine Veränderung der Lebenssituation an. Zu diesem Zeitpunkt zeigen sich auf Indikatorebene keine signifikanten Unterschiede mehr zwischen der unteren, mittleren und oberen sozialen Lage.
Aufgeschlüsselt nach den Einzelitems des Verhältnis-Empowerments zeigt sich in der Katamnese nach sechs Monaten, dass in der unteren Sozialschicht vor allem positive Veränderungen in sonstigen familiären Beziehungen (z. B. besseres Verhältnis zu Eltern oder Schwiegereltern $)^{1}$ häufiger vorgenommen wurden. Auch Veränderungen in der Beziehung zum Kind (z. B. ,reagiere ruhiger', ,mehr Zeit für uns zwei') und in der Partnerschaft (z.B. Aussprache mit Partner, endgültige Trennung vom Partner) sowie in beruflicher Hinsicht (z.B. Aufgabenverteilung geändert, Aufgabe des belastenden Jobs) werden in der unteren Sozialschicht signifikant häufiger angegeben. Lediglich hinsichtlich des Haushaltes (z. B. Unordnung besser ertragen können, bessere Einteilung der Hausarbeit) lassen sich keine bedeutsamen schichtspezifischen Unterschiede nachweisen. Nach zwölf Monaten gleichen sich die Soziallagen hinsichtlich der Häufigkeit der vorgenommenen Veränderungen an. Zu diesem Zeitpunkt werden in der unteren Soziallage nur noch Veränderungen in ,sonstigen familiären Beziehungen` signifikant häufiger angegeben.

\section{Erfolg des Verhältnis-Empowerments}

63,1\% der Mütter der oberen Soziallage geben in der ersten Nacherhebung nach sechs Monaten an, dass die Veränderung der Lebenssituation (eher) erfolgreich verlaufen ist. Die Erfolgseinschätzung in der unteren und mittleren Soziallage fällt nur geringfügig und nicht signifikant geringer aus ( $\odot$ Tab. 4 Zeile: ,Indikator-Variable Verhältnis-Empowerment'). Auch auf der Ebene der Einzelitems zeigt sich kein konsistenter Sozialgradient, nach dem die Erfolge von der oberen zur unteren Soziallage sukzessive abnehmen. Von den Frauen der oberen Soziallage werden in der Katamnese nach sechs Monaten vor allem Veränderungen der beruflichen Situation und der Organisation des Haushaltes überdurchschnittlich erfolgreich beurteilt. Demgegenüber werden Veränderungen der partnerschaftlichen Situation sowie im Freizeitbereich hier tendenziell kritischer bewertet. Die Mütter der mittleren Soziallage zeigen tendenziell größere Erfolge hinsichtlich Veränderungen im Freizeitbereich und familiärer Beziehungen, während Veränderungen vor allem in der Beziehung zum Kind, aber auch im Haushalt am negativsten beurteilt werden. Die Mütter der unteren Soziallage wei-

\footnotetext{
${ }^{1}$ Hierbei handelt es sich um typische Klartextangaben, die zu t3 von den Patientinnen zu den einzelnen Veränderungsbemühungen gemacht wurden. Siehe hierzu ausführlich [22].
} 
Tab. 4 Soziallagespezifische Erfolgseinschätzung der Einzelitems des Empowerments (in \%) zu t3 und t4.

\begin{tabular}{|c|c|c|c|c|c|c|c|c|c|c|c|}
\hline \multirow[b]{2}{*}{$\begin{array}{l}\text { Verhältnis- } \\
\text { Empowerment }\end{array}$} & \multicolumn{4}{|c|}{ Katamnese $\mathbf{t} 3$} & \multicolumn{4}{|c|}{ Katamnese t4 } & \multicolumn{3}{|c|}{$\begin{array}{l}\text { Übereinstimmung } \\
\text { t3-t4 in \% }\end{array}$} \\
\hline & $\begin{array}{l}\text { US } \\
n_{\max }=848\end{array}$ & $\begin{array}{l}\text { MS } \\
n_{\max }=1220\end{array}$ & $\begin{array}{l}\text { OS } \\
n_{\max }=390\end{array}$ & $\begin{array}{l}p\left(\mathrm{Chi}^{2}\right) \\
(\mathrm{df}=2)\end{array}$ & $\begin{array}{l}\text { US } \\
n_{\max }=747\end{array}$ & $\begin{array}{l}\text { MS } \\
n_{\max }=1174\end{array}$ & $\begin{array}{l}\text { OS } \\
n_{\max }=371\end{array}$ & $\begin{array}{l}p\left(C h i^{2}\right) \\
(d f=2)\end{array}$ & US & MS & os \\
\hline Indikatorvariable & 60,6 & 60,1 & 63,1 &, 570 & 57,2 & 59,8 & 60,6 & ,414 & 68,5 & 71,6 & 72,3 \\
\hline Partnerschaft & 52,2 & 47,4 & 45,6 & 187 & 47 & 46 & 42,7 &, 558 & 78,4 & 66,9 & 59,5 \\
\hline Beziehung Kind & 66,6 & 60 & 66,4 &, 020 & 68,6 & 72,6 & 75,4 &, 072 & 77,1 & 88,4 & 88 \\
\hline fam. Beziehungen & 50,3 & 54,1 & 52,1 & ,566 & 49,6 & 47,6 & 41,7 & , 155 & 65,6 & 70,6 & 58,3 \\
\hline berufliche Sit. & 52,6 & 58,2 & 67,3 & ,005 & 50 & 49,8 & 54,6 & ,409 & 70,1 & 71 & 72,9 \\
\hline Haushalt & 56,6 & 52,4 & 64,6 &, 014 & 51,1 & 47,1 & 50,6 & ,326 & 72 & 67,9 & 66,7 \\
\hline Freizeit & 57 & 59 & 54,2 & ,395 & 54 & 55,8 & 58,7 &, 443 & 68,9 & 66,7 & 66,7 \\
\hline $\begin{array}{l}\text { Verhaltens- } \\
\text { Empowerment }\end{array}$ & $\begin{array}{l}\text { US } \\
n_{\max }=1027\end{array}$ & $\begin{array}{l}\text { MS } \\
n_{\max }=1631\end{array}$ & $\begin{array}{l}\text { OS } \\
n_{\max }=501\end{array}$ & $\begin{array}{l}p\left(\mathrm{Chi}^{2}\right) \\
(\mathrm{df}=2)\end{array}$ & $\begin{array}{l}\text { US } \\
n_{\max }=760\end{array}$ & $\begin{array}{l}\text { MS } \\
n_{\max }=1220\end{array}$ & $\begin{array}{l}\text { OS } \\
n_{\max }=377\end{array}$ & $\begin{array}{l}p\left(C^{2} i^{2}\right) \\
(d f=2)\end{array}$ & US & MS & OS \\
\hline Indikatorvariable & 45,9 & 48,6 & 55,2 &, 002 & 45,1 & 44,2 & 47,8 & ,462 & 70,6 & 67,2 & 69,0 \\
\hline $\begin{array}{l}\text { kann mich besser } \\
\text { abgrenzen }\end{array}$ & 54,8 & 54,5 & 54,6 & ,991 & 59,4 & 57,3 & 60,1 &, 568 & 72,3 & 70,1 & 73,8 \\
\hline $\begin{array}{l}\text { nehme mir mehr } \\
\text { Zeit für mich }\end{array}$ & 47,5 & 51,5 & 54,1 & ,033 & 49,7 & 50,3 & 53,7 & ,423 & 67,5 & 66,4 & 65,9 \\
\hline $\begin{array}{l}\text { achte mehr auf } \\
\text { meine Gesundheit }\end{array}$ & 58,4 & 60,9 & 64,4 & ,082 & 55,1 & 56,9 & 64,2 & ,013 & 71,1 & 69,9 & 73,8 \\
\hline $\begin{array}{l}\text { suche mehr soziale } \\
\text { Unterstützung }\end{array}$ & 50 & 46,7 & 51,3 & ,126 & 47,3 & 44,9 & 50 & ,246 & 61,2 & 63 & 71,9 \\
\hline $\begin{array}{l}\text { kann eher über } \\
\text { Wut/Ärger sprechen }\end{array}$ & 57,4 & 55,2 & 63,6 & ,012 & 59,5 & 54,6 & 57,5 & ,140 & 71,4 & 70,3 & 72,2 \\
\hline $\begin{array}{l}\text { bin zufriedener } \\
\text { mit mir }\end{array}$ & 58,1 & 60,9 & 69 & $<, 001$ & 56,6 & 58,2 & 58,3 & ,778 & 71,1 & 72,6 & 68,2 \\
\hline $\begin{array}{l}\text { kann mich besser } \\
\text { entspannen }\end{array}$ & 37,6 & 36,1 & 46 & $<, 001$ & 37,4 & 31,6 & 37 & ,019 & 63,7 & 53,4 & 57,4 \\
\hline $\begin{array}{l}\text { fördere stärker } \\
\text { meine Gesundheit } \\
\text { aktiv }\end{array}$ & 52,3 & 55,2 & 63,4 & $<, 001$ & 51,2 & 51 & 61,8 & ,001 & 70,2 & 69,3 & 76,4 \\
\hline $\begin{array}{l}\text { kann Zeit besser } \\
\text { einteilen }\end{array}$ & 48,9 & 48,7 & 51,5 & ,596 & 45,6 & 43,1 & 46,8 & ,430 & 61,8 & 59,6 & 62,6 \\
\hline $\begin{array}{l}\text { kann besser mit } \\
\text { Belast. umgehen }\end{array}$ & 40,8 & 42,6 & 42,9 & ,618 & 40,9 & 39,8 & 40,3 & ,875 & 66,7 & 64,9 & 61,5 \\
\hline
\end{tabular}

sen überdurchschnittliche Erfolge hinsichtlich partnerschaftlicher Veränderungen auf, während berufliche Veränderungen hier am kritischsten bilanziert werden.

In der Katamnese nach zwölf Monaten fällt die Erfolgsbeurteilung soziallageübergreifend negativer aus. Lediglich der Aspekt ,Veränderungen in der Beziehung zum Kind“ erfährt bei allen Müttern, vor allem der oberen und mittleren Soziallage, eine deutlich positivere Beurteilung. Tendenziell bildet sich auch zu diesem Zeitpunkt eine höhere Erfolgseinschätzung der Mütter der oberen sozialen Lage ab. Auf der Ebene der Einzelitems lassen sich jedoch keine signifikanten Unterschiede zwischen den Soziallagen mehr ausmachen. Wie aus dem rechten Teil der - Tab. 4 ersichtlich wird, fällt die Übereinstimmung von t3 zu t4 bezüglich der Aspekte, Veränderungen der partnerschaftlichen Situation' sowie ,familiärer Beziehungen` insbesondere für die Mütter der oberen Soziallage vergleichsweise gering aus. So bestätigen nur 59,5\% bzw. 58,3\% der Mütter, die zu t3 von erfolgreichen Veränderungen in diesen Bereichen berichtet haben, dies auch zu t4.

\section{Erfolg des Verhaltens-Empowerments}

Bezüglich des Verhaltens-Empowerments lassen sich deutlich größere Erfolge bei den Müttern der oberen Soziallage feststellen ( $\bullet$ Tab. 4 Zeile: ,Indikator-Variable Verhaltens-Empower- ment'). Die Aufschlüsselung nach den einzelnen Empowermentbereichen ergibt, dass die Mütter der oberen Soziallage insbesondere die Aussagen, bin zufriedener mit mir und meinen Leistungen', ,kann mich besser entspannen“ sowie ,fördere stärker meine Gesundheit aktiv` signifikant häufiger bestätigen. Zwischen der mittleren und unteren Soziallage zeigen sich insgesamt nur moderate Differenzen. In der Katamnese nach zwölf Monaten geht insbesondere die Erfolgseinschätzung in der oberen, aber auch mittleren Soziallage zurück, während sie in der unteren Soziallage in etwa konstant bleibt. Bezüglich der Aussagen ,fördere stärker meine Gesundheit aktiv` und ,achte mehr auf meine Gesundheit' lässt sich nach wie vor eine höhere $\mathrm{Zu}$ stimmung in der oberen Soziallage nachweisen. Als wenig stabil von t3 zu t4 erwiesen sich soziallageübergreifend, hauptsächlich aber bei den Müttern der mittleren Soziallage vor allem die Aussagen ,kann mich besser entspannen“ sowie ,kann die Zeit besser einteilen".

\section{Sozialschichtspezifische Gesundheitsrelevanz von Empowerment}

Wie dem Modell 1 der Regressionsrechnung I ( $\bullet$ Tab. 5) zu entnehmen ist, hat die Höhe der psychischen Symptombelastung zu den vorangegangenen Untersuchungszeitpunkten soziallage- 
Tab. 5 Einfluss von Empowerment (Indikatorvariablen) auf die Höhe der psychischen Symptombelastung zu t3 und t4.

\begin{tabular}{|c|c|c|c|c|c|c|c|c|c|c|c|c|}
\hline \multirow[b]{3}{*}{ untere Soziallage } & \multicolumn{6}{|c|}{$\begin{array}{l}\text { Regressionsanalyse I: } \\
\text { Psychische Symptombelastung t3 }\end{array}$} & \multicolumn{6}{|c|}{$\begin{array}{l}\text { Regressionsanalyse II: } \\
\text { Psychische Symptombelastung t4 }\end{array}$} \\
\hline & \multicolumn{2}{|c|}{ Modell 1} & \multicolumn{2}{|c|}{ Modell 2} & \multicolumn{2}{|c|}{ Modell 3} & \multicolumn{2}{|c|}{ Modell 1} & \multicolumn{2}{|c|}{ Modell 2} & \multicolumn{2}{|c|}{ Modell 3} \\
\hline & Beta & $\mathbf{p}$ & Beta & $\mathbf{P}$ & Beta & p & Beta & p & Beta & p & Beta & p \\
\hline t1 Symptombelastung & ,29 & $<, 001$ &, 28 & $<, 001$ &, 27 & $<, 001$ &, 15 & ,006 &, 15 & ,003 &, 16 &, 002 \\
\hline t2 Symptombelastung &, 25 & $<, 001$ & ,18 & $<, 001$ & ,18 & $<, 001$ & ,18 &, 001 & ,15 & ,004 & , 14 & ,008 \\
\hline t3 Symptombelastung ${ }^{1}$ & & & & & & & 49 & $<, 001$ &, 45 & $<, 001$ & 42 &, 000 \\
\hline Verhaltens-Empowerment & & &,- 35 & $<, 001$ &,- 32 & $<, 001$ & & &,- 16 & $<, 001$ &,- 10 & ,046 \\
\hline Verhältnis-Empowerment & & & & &,- 07 & ,168 & & & & &,- 15 &, 002 \\
\hline Adjusted $R^{2}$ & & ,207 & ,320 & $11,3 \%)$ &, 322 & $11,5 \%)$ & & ,450 & & $(+2,1 \%)$ & & $(+3,7 \%)$ \\
\hline \multicolumn{13}{|l|}{ mittlere Soziallage } \\
\hline t1 Symptombelastung &, 27 & $<, 001$ &, 24 & $<, 001$ & ,26 & $<, 001$ &, 17 & $<, 001$ & ,19 & $<, 001$ & , 19 & $<, 001$ \\
\hline t2 Symptombelastung & ,30 & $<, 001$ &, 28 & $<, 001$ &, 23 & $<, 001$ & ,13 &, 001 & ,11 &, 015 & , 10 &, 017 \\
\hline t3 Symptombelastung ${ }^{1}$ & & & & & & &, 53 & $<, 001$ & ,47 & $<, 001$ &, 46 & $<, 001$ \\
\hline Verhaltens-Empowerment & & &,- 30 & $<, 001$ &,- 23 &, 001 & & &,- 16 & $<, 001$ &,- 13 & ,002 \\
\hline Verhältnis-Empowerment & & & & &,- 15 &, 001 & & & & &,- 08 & 038 \\
\hline Adjusted $\mathrm{R}^{2}$ & &, 224 & ,31 & $+8,6 \%)$ & ,326 & $10,2 \%)$ & &, 471 & & $(+2,1 \%)$ & & $+2,6 \%)$ \\
\hline \multicolumn{13}{|l|}{ obere Soziallage } \\
\hline t1 Symptombelastung & ,36 & $<, 001$ & ,37 & $<, 001$ & ,36 & $<, 001$ &, 21 &, 009 &, 21 &, 007 & ,21 & 007 \\
\hline t2 Symptombelastung & ,14 &, 034 & ,11 & $<, 001$ & ,11 & $<, 001$ &, 00 & ,958 &,- 01 & ,918 &, 00 & ,918 \\
\hline t3 Symptombelastung ${ }^{1}$ & & & & & & & ,42 & $<, 001$ &, 43 & $<, 001$ & ,43 &, 000 \\
\hline Verhaltens-Empowerment & & &,- 24 & $<, 001$ &,- 20 & ,002 & & &,- 15 &, 024 &,- 07 & 365 \\
\hline Verhältnis-Empowerment & & & & &,- 01 & ,129 & & & & &,- 18 &, 012 \\
\hline Adjusted $R^{2}$ & & 191 & ,24 & $+5,2 \%)$ & & $+6,3 \%)$ & & 293, & & $(+2,1 \%)$ &, 34 & $+4,7 \%)$ \\
\hline
\end{tabular}

Erläuterungen: Dargestellt werden die standardisierten Beta-Koeffizienten und die dazugehörige Irrtumswahrscheinlichkeit (p). Die Werte in Klammern unter ,Adjusted $\mathrm{R}^{2 *}$ geben ausgehend von Modell 1 den Zugewinn erklärter Varianz an. ${ }^{1}=$ nur für Regressionsrechnung t4.

übergreifend einen hohen Erklärungsanteil am Ausmaß der psychischen Symptombelastung zum Zeitpunkt t3. Die in das Modell 2 hinzugefügte Indikatorvariable des Verhaltens-Empowerments leistet soziallageübergreifend einen eigenständigen Beitrag zur signifikanten Erhöhung der Varianzaufklärung. Wie aus den Vorzeichen der Regressionskoeffizienten hervorgeht, führen erfolgreiche Empowermentprozesse erwartungsgemäß zu einer deutlichen Reduzierung der psychischen Symptombelastung. Mit einem Anteil erklärter Varianz von 11,3\% zeigt sich vor allem für die Mütter der unteren Soziallage ein hoher Gesundheitseffekt, während die Bedeutung für die Mütter der oberen Soziallage mit einem Varianzanteil von 5,2\% am geringsten ausfällt. Auf Itemebene zeigt sich, dass vor allem eine größere Selbstzufriedenheit mit geringeren psychischen Symptomen einhergeht ( 0 Tab. 6). Aber auch die Aussagen ,kann mich besser entspannen', ,kann besser mit Belastungen umgehen' sowie ,kann Zeit besser einteilen' weisen unabhängig von der Sozialschicht eine hohe Gesundheitsrelevanz auf. Für Mütter der unteren und mittleren Soziallage erweist sich zudem die Aussage , nehme mir mehr Zeit für mich' als besonders bedeutsam für die psychische Gesundheit.

Unter Hinzunahme des Verhältnis-Empowerments (Modell 3, - Tab. 5) geht der Einfluss des Verhaltens-Empowerments nur moderat zurück. Insgesamt kann im Modell 3 nur eine geringe Erhöhung der erklärten Varianz erzielt werden. So kann lediglich für die Mütter der mittleren Soziallage ein eigenständiger signifikanter Effekt des Verhältnis-Empowerments ermittelt werden, während vor allem für die Mütter der unteren Soziallage positive Veränderungen in der Lebenssituation - zumindest auf Indikatorebene - nur mit einer geringfügigen Verbesserung der psychischen Symptombelastung verknüpft sind. Auf der Ebene der Einzelitems zeigt sich, dass für die Mütter der mittleren Soziallage nahezu alle Aspekte des Verhältnis-Empower- ments relevant sind, vor allem Veränderungen der beruflichen Situation sowie Veränderungen in der Organisation des Haushalts ( $\bullet$ Tab. 6). Für die Mütter der unteren Soziallage geht der größte Einfluss von positiven Veränderungen der partnerschaftlichen Situation aus, während für die Mütter der oberen Soziallage Veränderungen im Beruf und Haushalt die größte Gesundheitsrelevanz besitzen.

Zu t4 (Regressionsrechnung II, $\odot$ Tab. 5) leistet die Höhe der psychischen Symptombelastung zu den vorangegangenen Untersuchungszeitpunkten aufgrund des zusätzlichen Zeitpunktes t3 einen noch höheren Erklärungsanteil an der Gesamtvarianz der psychischen Symptombelastung (Modell 1). Vor allem in der unteren und mittleren Soziallage wird hier mit Varianzaufklärungen von $45 \%$ bzw. $47 \%$ nahezu die Hälfte der Gesamtvarianz erklärt. Ähnlich wie bereits zu t3 fällt die durch das Modell 1 erklärte Varianz in der oberen Soziallage deutlich geringer aus. Auffällig ist hier, dass sich die psychische Symptombelastung zu t2 als kein bedeutsamer Prädiktor für den Zeitpunkt t4 erweist. Die Hinzunahme der Indikatorvariable zum Verhaltens-Empowerment führt zu einer signifikanten, jedoch nur moderaten Erhöhung der erklärten Varianz. Anders als zu t3 geht unter Hinzunahme des Verhältnis-Empowerments der Einfluss des Verhaltens-Empowerments signifikant zurück. Lediglich für die Mütter der mittleren Soziallage lässt sich im Beisein des Verhältnis-Empowerments auf Indikatorebene noch ein hochsignifikanter Einfluss des Verhaltens-Empowerment nachweisen. Auf Itemebene lassen sich soziallageübergreifend nach wie vor hochsignifikante Einflüsse hinsichtlich der Items ,bin zufriedener mit mir', ,kann besser mit Belastungen umgehen ' und ,kann mich besser entspannen' feststellen ( $\boldsymbol{0}$ Tab. $\mathbf{6})$. Ein höheren Effekt des Verhältnis-Empowerments lässt sich für die Mütter der oberen und unteren Soziallage ermitteln. Auf Itemebene ist von $\mathrm{t} 3$ zu t4 eine deutliche Verschiebung in der Gesund- 
Tab. 6 Einfluss von Empowerment (Einzelitems) auf die Höhe der psychischen Symptombelastung zu t3 und t4.

\begin{tabular}{|c|c|c|c|c|c|c|c|c|c|c|c|c|}
\hline \multirow[b]{3}{*}{ Verhältnis-Empowerment } & \multicolumn{6}{|c|}{ Psychische Symptombelastung $\mathrm{t} 3$} & \multicolumn{6}{|c|}{ Psychische Symptombelastung t4 } \\
\hline & \multicolumn{2}{|c|}{$\begin{array}{l}\text { US } \\
n_{\max }=257\end{array}$} & \multicolumn{2}{|c|}{$\begin{array}{l}\text { MS } \\
n_{\max }=334\end{array}$} & \multicolumn{2}{|c|}{$\begin{array}{l}\text { OS } \\
n_{\max }=148\end{array}$} & \multicolumn{2}{|c|}{$\begin{array}{l}\text { US } \\
n_{\max }=288\end{array}$} & \multicolumn{2}{|c|}{$\begin{array}{l}\text { MS } \\
\mathrm{n}_{\max }=366\end{array}$} & \multicolumn{2}{|c|}{$\begin{array}{l}\text { OS } \\
n_{\max }=159\end{array}$} \\
\hline & Beta & $\mathbf{p}$ & Beta & $\mathbf{p}$ & Beta & $\mathbf{p}$ & Beta & $\mathbf{p}$ & Beta & $\mathbf{p}$ & Beta & $\mathbf{p}$ \\
\hline Partnerschaft &,- 268 & $<, 001$ &,- 145 & ,010 &,- 151 & ,088 &,- 149 & ,014 &,- 167 & $<, 001$ &,- 223 &, 013 \\
\hline Beziehung Kind &,- 098 &, 074 &,- 155 &, 001 &,- 037 & ,622 &,- 091 &, 051 &,- 061 & ,152 &,- 040 & 600 \\
\hline fam. Beziehungen &,- 130 & ,082 &,- 235 & $<, 001$ &,- 154 & ,153 &,- 164 &, 004 &,- 135 &, 008 &,- 212 & 019 \\
\hline berufliche Situation &,- 153 &, 031 &,- 340 & $<, 001$ &,- 347 & 001 &,- 023 & ,701 &,- 130 &, 006 &,- 169 &, 040 \\
\hline Haushalt &,- 136 &, 043 &,- 272 & $<, 001$ &,- 364 & $<, 001$ &,- 158 &, 003 & 1,144 &, 004 &,- 183 &, 023 \\
\hline \multirow[t]{2}{*}{ Freizeit } &,- 111 &, 042 &,- 216 & $<, 001$ &,- 218 & ,018 &,- 158 &, 002 &,- 109 &, 012 &,- 087 & ,217 \\
\hline & \multicolumn{2}{|c|}{$\begin{array}{l}\text { US } \\
n_{\max }=496\end{array}$} & \multicolumn{2}{|c|}{$\begin{array}{l}\text { MS } \\
n_{\max }=666\end{array}$} & \multicolumn{2}{|c|}{$\begin{array}{l}\text { OS } \\
n_{\max }=307\end{array}$} & \multicolumn{2}{|c|}{$\begin{array}{l}\text { US } \\
n_{\max }=366\end{array}$} & \multicolumn{2}{|c|}{$\begin{array}{l}\text { MS } \\
n_{\max }=466\end{array}$} & \multicolumn{2}{|c|}{$\begin{array}{l}\text { OS } \\
n_{\max }=215\end{array}$} \\
\hline Verhaltens-Empowerment & Beta & $\mathbf{p}$ & Beta & $\mathbf{p}$ & Beta & $\mathbf{p}$ & Beta & $\mathbf{p}$ & Beta & $\mathbf{p}$ & Beta & $\mathbf{p}$ \\
\hline $\begin{array}{l}\text { kann mich besser } \\
\text { abgrenzen }\end{array}$ &,- 141 & $<, 001$ &,- 154 & $<, 001$ &,- 075 & ,177 &,- 147 & ,002 &,- 073 &, 082 & $<, 001$ & ,997 \\
\hline $\begin{array}{l}\text { nehme mir mehr Zeit für } \\
\text { mich }\end{array}$ &,- 206 & $<, 001$ &,- 216 & $<, 001$ &,- 158 &, 003 &,- 063 &, 173 &,- 082 &, 036 &, 044 &, 506 \\
\hline $\begin{array}{l}\text { achte mehr auf meine } \\
\text { Gesundheit }\end{array}$ &,- 146 & $<, 001$ &,- 198 & $<, 001$ &,- 172 &, 001 &,- 069 & ,131 &,- 097 &, 013 &,- 117 & ,070 \\
\hline $\begin{array}{l}\text { suche mehr soziale Unter- } \\
\text { stützung }\end{array}$ &,- 171 & $<, 001$ &,- 085 & ,019 &,- 009 &, 878 &,- 029 &, 556 &,- 060 & , 140 &,- 106 & 129 \\
\hline $\begin{array}{l}\text { kann eher über Wut/Ärger } \\
\text { sprechen }\end{array}$ &,- 185 & $<, 001$ &,- 168 & $<, 001$ &,- 123 & ,036 &,- 108 &, 023 &,- 125 &, 002 &,- 074 & ,310 \\
\hline bin zufriedener mit mir &,- 286 & $<, 001$ &,- 302 & $<, 001$ &,- 255 & $<, 001$ &,- 208 & $<, 001$ &,- 175 & $<, 001$ &,- 277 & $<, 001$ \\
\hline $\begin{array}{l}\text { kann mich besser ent- } \\
\text { spannen }\end{array}$ &,- 255 & $<, 001$ &,- 187 & $<, 001$ &,- 228 & $<, 001$ &,- 121 &, 010 &,- 114 &, 003 &,- 199 &, 002 \\
\hline $\begin{array}{l}\text { fördere stärker meine } \\
\text { Gesundheit aktiv }\end{array}$ &,- 161 & $<, 001$ &,- 126 & $<, 001$ &,- 125 &, 022 &,- 062 & ,203 &,- 046 & ,243 &,- 085 & ,197 \\
\hline kann Zeit besser einteilen &,- 201 & $<, 001$ &,- 248 & $<, 001$ &,- 215 & $<, 001$ &,- 120 &, 013 &,- 125 &, 004 &,- 096 & ,831 \\
\hline $\begin{array}{l}\text { kann besser mit Bela- } \\
\text { stungen umgehen }\end{array}$ &,- 251 & $<, 001$ &,- 287 & $<, 001$ &,- 194 & $<, 001$ &,- 212 & $<, 001$ &,- 167 & $<, 001$ &,- 174 & ,006 \\
\hline
\end{tabular}

Erläuterungen: Ergebnisse univariater Regressionsanalysen, kontrolliert für die jeweils vorausgegangene psychische Symptombelastung (für $\mathrm{t} 3=\mathrm{t} 1 \mathrm{und} \mathrm{t} 2$, für $\mathrm{t} 4=\mathrm{t} 1$, $\mathrm{t} 2$ und $\mathrm{t3}$ ). US = untere Soziallage, MS = mittlere Soziallage, OS= obere Soziallage. Dargestellt werden die standardisierten Beta-Koeffizienten und die dazugehörige Irrtumswahrscheinlichkeit (p).

heitsrelevanz der Lebensbereiche zu beobachten. Einen Bedeutungszuwachs zu t4 erfahren vor allem Veränderungen in ,sonstigen familiären Beziehungen'. Insgesamt lässt sich zu t4 ein deutlicher Bedeutungsverlust von Empowerment beobachten.

\section{Diskussion \\ $\nabla$}

Sozialschichtspezifische kurz-, mittel- und langfristige Therapieeffekte

Die grundsätzliche Effektivität von Vorsorge- und Rehabilitationsmaßnahmen für Mütter/Väter und ihre Kinder auf den Dimensionen ,somatische‘, ,psychische‘ sowie ,funktionale' Gesundheit ist inzwischen hinreichend belegt. Analog zu den Evaluationsbefunden aus der fachspezifischen Rehabilitation [29] weisen die Befunde auf hohe kurzfristige Erfolge der Maßnahme hin, die auf einem mittleren Niveau auch noch nach der Rückkehr nach Hause nachweisbar sind [14,30]. Mit der vorliegenden Arbeit wurde eine Differenzierung dieser Befunde in Abhängigkeit von der sozialen Lebenssituation der Patientinnen vorgenommen. Eine derartige Perspektivenerweiterung um den Aspekt ,soziale Ungleichheit" findet im Rahmen der Versorgungsforschung zunehmend statt. Bislang konzentrieren sich die Arbeiten jedoch weitestgehend auf soziallagespezifische Unterschiede in der Inanspruchnahme des Gesundheitswesens [Überblick siehe 18]. Die wenigen auf der ,Outcome‘-Seite vorlie- genden Befunde sind nicht einheitlich. So weisen Backmund et al. [zitiert in 18] auf geringere Erfolge in der Suchtbehandlung bei bildungsbenachteiligten Patienten hin, Deck [31] konnte hingegen bezüglich der rehabilitativen Erfolge keine soziallagespezifische Benachteiligung ermitteln. Die vorliegende Arbeit hat gezeigt, dass sozial benachteiligte Mütter kurzfristig, d.h. unmittelbar am Ende der Maßnahme die höchsten Therapieeffekte in der Reduktion psychischer Symptome aufweisen. Dieser Befund ist bemerkenswert, gleichwohl er dem größeren Veränderungspotenzial in der unteren Soziallage aufgrund der signifikant höheren psychischen Symptombelastung entspricht. Er verdeutlicht, dass Mütter aus benachteiligten Soziallagen am unmittelbaren Maßnahmenende nicht nur vergleichbar, sondern überdurchschnittlich von der Intervention profitieren können. Zu beachten ist hierbei jedoch, dass der statistische Effekt der ,Regression zur Mitte، [32] zur Reduktion der psychischen Symptombelastung in der unteren Soziallage beigetragen haben könnte, da hier zum Zeitpunkt t1 überdurchschnittlich viele Mütter extreme Werte angegeben haben. Die kurzfristig erzielten Reduktionen psychischer Symptome gehen bei den Müttern der unteren, aber auch mittleren Soziallage nach der Rückkehr in den häuslichen Alltag überdurchschnittlich stark zurück. Die Folge ist, dass sich die gesundheitliche Ungleichheit sechs Monate nach der Maßnahme wieder vergrößert. Nach weiteren sechs Monaten ist eine erneute Annäherung zwischen den Soziallagen zu verzeichnen. Insgesamt lässt sich damit fest- 
halten, dass die Maßnahme zu einer Verbesserung des psychischen Gesundheitszustandes (auch) sozial benachteiligter Mütter geführt hat, jedoch nicht zur Verringerung gesundheitlicher Ungleichheit in diesem Bereich.

\section{Soziallagespezifischer Erfolg und Gesundheitseffekt von Empowerment}

Im Rahmen der vorliegenden Arbeit wurde postuliert, dass dem Empowerment eine zentrale Bedeutung für die poststationäre Entwicklung der psychischen Symptombelastung zukommt. Es wurde angenommen, dass die kurzfristig erzielten Therapieeffekte auch nach der Rückkehr in den häuslichen Alltag aufrechterhalten werden können, wenn es den Frauen im Sinne des Empowerments gelingt, ihre kleinräumige Lebenssituation positiv zu verändern bzw. die während der Maßnahme erlernten Verhaltensänderungen und Kompetenzen im Alltag umzusetzen. Ein derartiger Empowerment-Effekt auf die Nachhaltigkeit der Therapieerfolge konnte bereits ermittelt werden [22]. Unklar war bislang jedoch, ob sich der Erfolg und die Gesundheitsrelevanz von Empowerment in Abhängigkeit von der sozialen Lage der Patientinnen unterscheiden.

Entgegen den Erwartungen haben die vorliegenden Ergebnisse zunächst gezeigt, dass sozial benachteiligte Mütter prinzipiell gut für Empowermentziele motivierbar waren und nach der Rückkehr in den häuslichen Alltag auch häufiger Veränderungen der Lebenssituation vorgenommen haben. Auch wiesen die vorliegenden Befunde zum Erfolg des Verhältnis-Empowerments keinen Schichtgradienten auf. Vielmehr gaben in etwa gleich viele Mütter aus der unteren, mittleren und oberen Soziallage an, dass Veränderungen der Lebenssituation erfolgreich verlaufen sind. Die Analysen zur Gesundheitsrelevanz von Empowerementprozessen haben ergeben, dass das Verhältnis-Empowerment vor allem in der Katamnese nach sechs Monaten (t3) insgesamt nur einen moderaten Einfluss auf die poststationäre Entwicklung der psychischen Symptombelastung ausübt. Der ermittelte relative Bedeutungszuwachs von t3 zu t4 könnte darauf hindeuten, dass Veränderungen der Lebenssituation erst langfristig ihre Wirkung auf die psychische Gesundheit entfalten. Insgesamt ging vom Verhältnis-Empowerment entgegen den Erwartungen jedoch kein bedeutsamer Gesundheitseffekt aus. Kritisch anzumerken ist in diesem Zusammenhang, dass sich die Befunde zum Verhältnis-Empowerment insgesamt als unzureichend konsistent erwiesen. Davon zeugt, dass die prioritär gesundheitsrelevanten Lebensbereiche zwischen den beiden Katamnesezeitpunkten $\mathrm{t} 3$ und $\mathrm{t} 4$ in allen Soziallagen deutlich variierten. Insbesondere die Angaben zu t4 erscheinen aufgrund der relativ großen zeitlichen Entfernung zur Intervention (12 Monate) hinsichtlich ihrer Validität als problematisch. Aus diesem Grund sollten die Befunde zum Verhältnis-Empowerment mit der entsprechenden Vorsicht interpretiert werden.

Als wesentlich bedeutsamer für nachhaltige Entwicklung der psychischen Gesundheit erwiesen sich in der vorliegenden Untersuchung positive Entwicklungen hinsichtlich des Aufbaus gesundheitsförderlicher Verhaltensweisen, Einstellungen und Kompetenzen im Sinne des Verhaltens-Empowerments. Insbesondere für die Mütter der unteren und mittleren Soziallage konnte eine hohe Gesundheitsrelevanz ermittelt werden. Der hohe theoretische Stellenwert von Empowerment für die Gesundheitsförderung sozial Benachteiligter konnte damit in der vorliegenden Untersuchung für den Aspekt ,Verhaltens-Empowerment' empirisch untermauert werden. Die individuellen Möglichkeiten des Aufbaus von personalen Ressourcen und
Kompetenzen erwiesen sich jedoch als ein sozial ungleich verteiltes Gut. So wiesen die Mütter der oberen Soziallage deutlich höhere Empowerment-Erfolge auf, während sich die Erfolge zwischen der unteren und mittleren Soziallage nicht wesentlich unterschieden. Insbesondere die Empowermentaspekte ,bin zufriedener mit mir und meinen Leistungen', ,fördere stärker meine Gesundheit aktiv، sowie ,kann mich besser entspannen` wurden von Müttern der oberen Soziallage signifikant positiver beurteilt. Dies entspricht den bisherigen Befunden, die auf optimalere Empowermentbedingungen in der oberen Soziallage hinweisen $[9,10,12]$. Die in der Katamnese nach sechs Monaten ermittelte geringere Nachhaltigkeit der Therapieeffekte in der unteren und mittleren Soziallage (s. o.) lässt sich damit - zumindest zum Teil - auf geringere poststationäre Erfolge im Verhaltens-Empowerment zurückführen. Gleichzeitig weisen die Befunde zur hohen Gesundheitsrelevanz von Empowerment insbesondere für weniger privilegierte Soziallagen darauf hin, dass Empowermentprozesse prinzipiell zu einer Reduzierung gesundheitlicher Ungleichheit beitragen können. Die Voraussetzung hierfür ist jedoch, dass die Empowermenterfolge für weniger privilegierte Mütter optimiert werden. Wirkungsvoll für die Verringerung gesundheitlicher Ungleichheit bezüglich des Outcomes ,psychische Symptombelastung، erscheinen vor allem Bemühungen, die Selbstzufriedenheit benachteiligter Mütter langfristig zu erhöhen. Denn hierbei handelt es sich um den Empowermentaspekt, der die höchste Bedeutung für die nachhaltige Stabilisierung der psychischen Gesundheit aufwies, gleichzeitig jedoch von den Müttern der unteren und mittleren Soziallage deutlich negativer bilanziert wurde.

Grundsätzlich hat sich gezeigt, dass die Gesundheitsrelevanz von Empowerment zwölf Monate nach der Maßnahme deutlich nachlässt. Damit lässt sich festhalten, dass Empowemententwicklungen vor allem in den ersten sechs Monaten nach der Maßnahme gesundheitsrelevant sind. Die deutliche Abnahme der Gesundheitseffekte in der Katamnese nach zwölf Monaten weist darauf hin, dass poststationäre Maßnahmen erforderlich sind, um den Gesundheitseffekten eine größere Nachhaltigkeit zu verleihen.

\section{Grenzen der sozialschichtspezifischen Betrachtung}

In der Literatur zum Thema ,Gesundheitliche Ungleichheit und Gender" wird zunehmend auf die Bedeutung weiterführender Ungleichheitsdimensionen von Frauen, insbesondere der familiären Situation und der Vereinbarkeit von Beruf und Familie verwiesen [23-25]. Die vorliegende Arbeit verfolgte das Ziel, zunächst den Einfluss struktureller Benachteiligung und damit der ,klassischen' Sozialindikatoren auf die Entwicklung der psychischen Gesundheit und der Empowermentprozesse zu analysieren. Aus diesem Grund wurden an dieser Stelle nicht die clusteranalytisch ermittelten sieben differenzierten Lebenslagen, sondern ausschließlich die drei übergeordneten Sozialschichten betrachtet. Unter dem Strich zeigte sich, dass die Mütter der oberen Soziallage gemäß ihrer Statusposition zumeist überdurchschnittliche Gesundheitschancen und Empowermenterfolge aufwiesen. Der Erfolg in der unteren und mittleren Soziallage gestaltete sich jedoch weniger ,schichtspezifisch'. So wiesen die Mütter der mittleren Soziallage gegenüber der unteren Soziallage nicht durchweg bessere Gesundheits- und Empowermentchancen auf und insgesamt ließen sich nur moderate Unterschiede zwischen den beiden Soziallagen nachweisen. Dies deutet darauf hin, dass die schichtspezifische Analyse im vorliegenden Fall nur begrenzt aussagekräftig ist. Aufschlussreich er- 
scheint vor diesem Hintergrund eine differenzierte Betrachtung mütterspezifischer Lebenslagen, wie sie in dem hier zugrunde gelegten clusteranalytischen Design der Soziallagebestimmung bereits angelegt ist.

\section{Restriktionen}

Einschränkungen in der Aussagekraft der ermittelten Befunde ergeben sich vor allem durch das Fehlen einer Kontrollgruppe. Die in der vorliegenden Arbeit ermittelten nachhaltigen Verbesserungen des Gesundheitszustandes sowie die Empowermenteffekte können letztendlich nicht eindeutig auf die stattgefundene Intervention zurückgeführt werden, da auch zahlreiche andere Einflüsse bzw. Zufallseffekte diese Veränderungen bewirkt haben könnten. Um dieses Problem zu minimieren, wurde in der Instruktion zu den Empowerment-Items explizit der Bezug zur Maßnahme hergestellt. Letztendlich kann die Frage der Rückführbarkeit auf die Intervention jedoch nur im Rahmen eines kontrollierten und randomisierten Studiendesigns geklärt werden. Begrenzungen in der Aussagekraft ergeben sich zudem durch das retrospektive Erhebungsdesign zur Messung von Empowerment, welches keine Aussagen zu Entwicklungsprozessen im Zeitverlauf zulässt. Für zukünftige Analysen anstrebenswert wären vor diesem Hintergrund längsschnittlich angelegte Mehrpunkt-Erhebungen, die einen prä-post-Vergleich ermöglichen.

\section{Literatur}

1 Bundeszentrale für gesundheitliche Aufklärung (BZgA). Kriterien guter Praxis in der Gesundheitsförderung bei sozial Benachteiligten. Ansatz - Beispiele - Weiterführende Informationen. Köln; 2006

2 Stark $W$. Empowerment. Neue Handlungskompetenzen in der psychosozialen Praxis. Freiburg im Breisgau: Lambertus Verlag; 1996

3 Herriger N. Empowerment in der sozialen Arbeit. Eine Einführung. 3. erweiterte und aktualisierte Auflage. Stuttgart: Verlag W. Kohlhammer; 2006

4 Rissel C. Empowerment: the holy grail of health promotion? Health Promotion International 1994; 9: 39-47

5 Loss J, Eichhorn C, Gehlert J et al. Gemeindenahe Gesundheitsförderung - Herausforderung an die Evaluation. Gesundheitswesen 2007; 69: 77-87

6 Kolip P. Ressourcen für Gesundheit - Potenziale und ihre Ausschöpfung. Gesundheitswesen 2003; 65: 155-162

7 Rosenbrock R. Prävention und Gesundheitsförderung - gesundheitswissenschaftliche Grundlagen für die Politik. Gesundheitswesen 2004; 66: 146-152

8 Klaes L, Troschke Jv. Die Deutsche Herz-Kreislauf-Präventionsstudie. Berlin: Hans Huber; 1998

9 Keupp H. Gesundheit als Lebenssouveränität - Ein sozial ungleich verteiltes Gut. Störfaktor, Zeitschrift kritischer Psychologinnen und Psychologen 1995; 31: 5-28

10 Nestmann F. Soziale Gerechtigkeit und Empowerment. Perspektiven des gemeindepsychologischen Modells. Archiv für Wissenschaft und Praxis der sozialen Arbeit 1999; 30: 129-150

11 Scheffer S, Dauven S, Sieverding M. Soziodemografische Unterschiede in der Teilnahme an Krebsfrüherkennungsuntersuchungen (KFU) in Deutschland - Eine Übersicht. Gesundheitswesen 2006; 68: 139-146

12 Pflaumer E. Der Widersprüchlichkeit Aufmerksamkeit schenken - Empowerment als Denk- und Handlungsansatz in der Gesundheitsförderung. In: Miller T, Pankofer S, Hrsg. Empowerment konkret! Handlungsentwürfe und Reflexionen aus der psychosozialen Praxis. Stuttgart: Lucius \& Lucius; 2000; 63-77
13 Wallerstein $N$. What is the evidence on effectiveness of empowerment to improve health? Copenhagen, WHO Regional Office for Europe. Health Evidence Network report; 2006; http://www.euro.who.int/ Document/E88086.pdf

14 Arnhold-Kerri S, Sperlich S, Collatz J. Krankheitsprofile und Therapieeffekte von Patientinnen in Mutter-Kind-Einrichtungen. Rehabilitation 2003; 42: 290-299

15 Collatz J, Fischer G, Thies-Zajons S. Mütterspezifische Belastungen - Gesundheitsstörungen - Krankheit. Berlin: Verlag für Wissenschaft und Bildung; 1998

16 Strittmatter $R$, Bengel J, Brombacher $C$ et al. Die Inanspruchnehmerinnen von Mutter-Kind-Kuren. Rehabilitation 1997; 36: 176184

17 Neitemeier S. Müttergenesungskuren - Ihre Ziele und ihre Wirksamkeit. Theorie und Praxis der sozialen Arbeit 1994; 5: 192-195

18 Janßen C, Grosse Frie K, Ommen O. Der Einfluss sozialer Ungleichheit auf die medizinische und gesundheitsbezogene Versorgung in Deutschland. In: Richter M, Hurrelmann K, Hrsg. Gesundheitliche Ungleichheit. Grundlagen, Probleme, Perspektiven. Wiesbaden: VS Verlag für Sozialwissenschaften; 2006; 141-156

19 Franke G H. SCL-90-R. Die Symptom-Checkliste von Derogatis - Deutsche Version. Göttingen: Beltz Test GmbH, 2. Auflage; 2002

20 Brähler E, Klaghofer R. Konstruktion und teststatistische Prüfung einer Kurzform der SCL-90-R. Zeitschrift für klinische Psychologie, Psychiatrie und Psychotherapie 2001; 49: 115-124

21 Collatz J, Borchert H, Brandt A et al. (1994): Effektivität, Bedarf und Inanspruchnahme von medizinischen und psychosozialen Versorgungseinrichtungen für Frauen und Mütter mit Kindern. Der Beitrag von Mütterkuren zur Frauengesundheit. Stuttgart: Kohlhammer; 1993

22 Sperlich S. Lässt sich die nachhaltige Verbesserung des psychischen Befindens von Müttern nach einer mütterspezifischen Vorsorge- und Rehabilitationsmaßnahme durch Empowerment erklären? Praxis Verhaltenstherapie. Klinische Verhaltensmedizin und Rehabilitation 2006; 72: 148-158

23 Östlin P. Gender perspective on socioeconomic inequalities in health. In: Mackenbach J, Bakker M, Hrsg. Reducing inequalities in health. A european perspective. London and New York, Routledge; 2002; 315-324

24 Sacker A, Firth D, Fitzpatrick $R$ et al. Comparing health inequality in men and women: prospective study of mortality 1986-96. British Medical Journal 2000; 320: 1303-1307

25 Babitsch B. Soziale Ungleichheit, Geschlecht und Gesundheit. Bern: Verlag Hans Huber; 2005

26 Bacher J. Clusteranalyse: Anwendungsorientierte Einführung. München: Oldenburg Verlag; 1994

27 Babitsch B. Soziale Ungleichheit und Gesundheit bei Frauen in Westdeutschland. In: RKI-Schriften 1998; 1: 95-112

28 Cohen J. Quantitative methods in psychology. A power primer. Psychological Bulletin 1992; 112: 155-159

29 Gerdes N, Weidemann H, Jäckel WH. Die Protos-Studie. Ergebnisqualität stationärer Rehabilitation in 15 Kliniken der Wittgensteiner Kliniken-Allianz (WKA). Darmstadt: Steinkopff Verlag; 2000

30 Meixner M, Glattacker M, Gerdes N et al. Behandlungseffekte in MutterKind-Einrichtungen - Ergebnisse des externen Qualitätsmanagements. Rehabilitation 2001; 40: 280-288

31 Deck $R$. Soziale Ungleichheit in der medizinischen Rehabilitation. Abstractband zum Kongress Medizin und Gesellschaft. Mönchengladbach: Rheinware Verlag; 2007; 5-6

32 Zwingman C, Wirtz M. Regression zur Mitte. Methoden in der Rehabilitationsforschung 2005; 44: 244-251 